\title{
FONOAUDIOLOGIA E BULLYING: AÇÃO DE PROMOÇÃO DE SAÚDE NA ESCOLA
}

\author{
FONOAUDIOLOGÍA Y BULLYING: ACCIÓN DE PROMOCIÓN DE LA SALUD EN LA \\ ESCUELA
}

\author{
SPEECH, LANGUAGE AND HEARING SCIENCES AND BULLYING: HEALTH \\ PROMOTION ACTION AT SCHOOL
}

\author{
Fernanda Yasmin Odila Maestri Miguel PADILHA ${ }^{1}$ \\ Anna Carolina Goulart RODRIGUES ${ }^{2}$ \\ Isabela Costa SILVEIRA ${ }^{3}$ \\ Aline Megumi ARAKAWA-BELAUNDE ${ }^{4}$
}

RESUMO: Objetivo: O objetivo deste artigo foi descrever a realização de uma ação fonoaudiológica na escola como uma possibilidade de promover a saúde em relação à temática bullying, na busca pelo respeito às diferenças. Método: $\mathrm{O}$ trabalho foi realizado com as crianças do $1^{\circ}$ e $2^{\circ}$ ano do ensino fundamental, que estudavam no período matutino na maior escola da América Latina. A ação foi realizada por meio da prática teatral, em consonância com as políticas públicas de saúde vigentes. Resultados: Observou-se que o teatro despertou o interesse das crianças que por sua vez reagiram de forma positiva diante discussões que surgiram posteriormente à apresentação do grupo. Além disso pode-se observar o envolvimento dos professores presentes no momento bem como da coordenação da escola. Conclusões: a fonoaudiologia mostrou-se aliada na promoção da saúde bem como no trabalho intersetorial, com a educação.

PALAVRAS-CHAVE: Fonoaudiologia. Promoção da saúde. Serviços de saúde escolar. Atenção primária à saúde.

RESUMEN: Objetivo: El objetivo de este artículo fue describir la realización de una acción fonoaudiológica en la escuela como una posibilidad de promover la salud en relación a la temática de bullying, en la búsqueda por el respeto a las diferencias. Método: El trabajo fue realizado con los niños del $1^{\circ}$ y $2^{\circ}$ año de la enseñanza fundamental, que estudiaban en el período matinal en la mayor escuela de América Latina. La acción fue realizada por medio de la práctica teatral, en consonancia con las políticas públicas de salud vigentes. Resultados: Se observó que el teatro despertó el interés de los niños que a su vez reaccionaron de forma positiva ante discusiones que surgieron posteriormente a la presentación del grupo. Además, se pudo observar la implicación de los profesores presentes

${ }^{1}$ Universidade Federal de Santa Catarina (UFSC), Florianópolis - SC - Brasil. Discente do Departamento de Fonoaudiologia. ORCID: <https://orcid.org/0000-0003-4780-5423>. E-mail: fernandaayasmin@gmail.com ${ }^{2}$ Universidade Federal de Santa Catarina (UFSC), Florianópolis - SC - Brasil. Discente do Departamento de Fonoaudiologia. ORCID: <https://orcid.org/0000-0001-6680-9919>. E-mail: annagoulart15@gmail.com

${ }^{3}$ Universidade Federal de Santa Catarina (UFSC), Florianópolis - SC - Brasil. Discente do Departamento de Fonoaudiologia. ORCID: <https://orcid.org/0000-0001-9104-6458>. E-mail: isabelasilveira_@hotmail.com

${ }^{4}$ Universidade Federal de Santa Catarina (UFSC), Florianópolis - SC - Brasil. Docente do Departamento de Fonoaudiologia. ORCID: <https://orcid.org/0000-0002-2159-6486>. E-mail: arakawa.aline@ufsc.br 
en el momento así como de la coordinación de la escuela. Conclusiones: la fonoaudiología se mostró aliada en la promoción de la salud así como en el trabajo intersectorial, con la educación.

PALABRAS CLAVE: Fonoaudiología. Promoción de la salud. Servicios de salud escolar. Atención primaria de salud.

ABSTRACT: Purpose: The aim of this study was to describe the realization of a speech language and hearing sciences action in school as an opportunity to promote health related to bullying theme, in the quest for respect for differences. The work was carried out with the children of the 1st and 2nd year of primary school, who studied in the morning in the largest school in Latin America. The action was carried out through the theater practice, in line with the current health policies. Results: It was observed that the theater aroused the children interest that responded positively to discussions that emerged after group presentation. Furthermore, it was observed the teachers and school coordinator involvement. Conclusions: the speech language and hearing sciences proved be and ally in the health promotion as well as with intersectoral work, with the education.

KEYWORDS: Speech. language and hearing sciences. Health promotion. School health services. Primary health care.

\section{Introdução}

O Bullying (do inglês bully = valentão, brigão; sem tradução adequada ao português) refere-se a atitudes agressivas, intencionais e repetidas, que acontecem sem um motivo aparente cometidas por um estudante ou mais contra outro(s), causando dor e angústia, sendo executadas dentro de uma relação desigual de poder (MALTA, 2010).

O bullying é classificado como direto ou indireto. O direto se refere aos apelidos, agressões físicas, ameaças, roubos, ofensas verbais ou expressões e gestos que causam desconforto aos alvos, sendo mais cometidos por meninos. O bullying indireto é caracterizado por atitudes de indiferença, isolamento, difamação, sendo esse cometido com maior frequência pelas meninas (FEKKES; PIJPERS; VERLOOVE-VANHORICK, 2005).

É importante que a temática seja inserida no currículo de aprendizagem escolar além dos conteúdos programáticos. Além de promover o respeito como uma atitude primordial, deve-se mostrar por meio de diálogos e práticas o valor do outro, fazer com que a criança se sinta bem em respeitar e ajudar seus colegas. Com isso, as políticas públicas de atenção a população que promovam ações preventivas ao bullying e também a violência no geral são extremamente necessárias nas escolas (MAZINI; BRANCO, 2012). 
Tendo em vista a necessidade de ações de promoção e prevenção da saúde nas escolas, o Ministério da Saúde junto ao Ministério da Educação desenvolveu o Programa Saúde na Escola (PSE), criado sob o Decreto $\mathrm{n}^{\circ}$ 6.286, de 5 de dezembro de 2007 (FIGUEIREDO; MACHADO; ABREU, 2010). Seu objetivo é contribuir para a formação integral dos estudantes da rede pública de educação básica por meio de ações de prevenção, promoção e atenção à saúde. Para isso, é preciso que as equipes de saúde da família e a escola trabalhem juntas, para fortalecer a atenção integral aos estudantes. Dentre as ações contempladas pelo PSE encontra-se o desenvolvimento de estratégias de atenção à saúde da comunicação humana no ambiente escolar, o qual estimula o conhecimento sobre cuidados com a saúde, gerando melhor qualidade de vida para a comunidade (JACOÉ et al., 2014).

O PSE está contemplado nas ações vinculadas à atenção básica, que segundo Portaria MS/GM n 2.488, de 21 de outubro de 2011, caracteriza-se por um conjunto de ações de saúde, individual e coletivo, relacionadas à promoção e proteção da saúde, prevenção de agravos, diagnóstico, tratamento, reabilitação, redução de danos e manutenção da saúde com o objetivo de desenvolver uma atenção integral que impacte na saúde e autonomia dos indivíduos e nos determinantes e condicionantes de saúde das coletividades (BRASIL, 2011a).

A atenção básica é um dos campos de atuação do profissional fonoaudiólogo bem como o ambiente escolar. Neste âmbito o mesmo busca desenvolver, em parceria com professores, ações que contribuam para a promoção e prevenção de alterações fonoaudiológicas e que beneficiem e aperfeiçoem o ensino e aprendizagem de um maior número de crianças (OLIVEIRA; NATAL, 2011).

A inserção da Fonoaudiologia na atenção básica ocorreu nos anos de 1970 a 1980, através das Secretarias de Educação e de Saúde (BEFI, 1997). A ação preventiva que sugere a promoção de ações na escola, realizadas pelo fonoaudiólogo junto a pais e professores, está relacionada aos esclarecimentos e discussões acerca dos aspectos de normalidade sobre a fala, audição, voz, entre outros. Com os alunos, compete ao fonoaudiólogo, identificar, através da aplicação de exames, as dificuldades na compreensão de ordens, na emissão oral, respiração e voz. Além disso, um programa de atuação fonoaudiológica nas escolas, deve considerar alguns aspectos, como a situação cultural e social da escola e seus alunos, a motivação dos pais, direção e professores e a existência da integração entre o trabalho realizado na escola e em âmbito familiar (VIEIRA et al., 2004; PEREIRA; SANTOS; OSBORN, 2004).

Ressalta-se como um pilar importante nas ações envolvendo a temática do bullying aquelas que tratam da promoção da saúde e os determinantes sociais da saúde. A promoção da 
saúde vem ao encontro com o proposto pela portaria $\mathrm{n}^{\circ} 2.446$, de 11 de novembro de 2014 , que revisou a política criada em 2006 a qual contempla a temática da inclusão social (BRASIL, 2014).

A Política Nacional de Promoção da Saúde traz o conceito ampliado de saúde junto ao desenvolvimento de estratégias e formas de produzir saúde, no âmbito individual e coletivo, caracterizando-se pela articulação e cooperação intra e intersetorial. Além disso, em seu art. $3^{\circ}$, traz como valor fundante a inclusão social, ao garantir o acesso aos benefícios da vida em sociedade para os indivíduos de forma equânime e participativa, buscando reduzir as iniquidades (BRASIL, 2014).

Neste contexto, cabe salientar a criação do Plano Nacional dos Direitos da Pessoa com Deficiência - Plano Viver sem Limite, instituído sob o decreto $n^{\circ} 7.612$ de 17 de novembro de 2011. Essa política tem como propósito promover o exercício dos direitos da pessoa com deficiência, através da integração e articulação de políticas, programas e ações. Os eixos de ação do Plano Viver sem Limite envolvem a atenção à saúde, inclusão e acessibilidade (BRASIL, 2011b).

A articulação das políticas em prol de um objetivo em comum impulsiona a ação intersetorial mediante a parceria estabelecida entre a educação e saúde, sendo a escola um ambiente que proporciona o aumento das possibilidades de acesso a atividades educativas, culturais, esportivas e lazer, por fim, gerando condições para a promoção da saúde (SANTOS et al., 2012).

Dado o que se precede, o presente estudo teve como objetivo apresentar um relato de experiência de uma ação fonoaudiológica na escola como uma possibilidade de promover a saúde em relação à temática bullying, em consonância com as políticas públicas de saúde vigentes, através da prática teatral.

\section{Materiais e métodos}

Trata-se do relato de experiência, vivenciado no segundo semestre de 2015, vinculada a uma disciplina da sexta fase do curso de fonoaudiologia de uma Universidade do sul do país. As ações estavam relacionadas à promoção e manutenção da saúde e prevenção de doenças no âmbito escolar.

Através de ações educativas que visam o conhecimento e a conscientização, buscou-se promover a saúde e incentivar práticas de vida saudáveis. Nesse contexto a escola assume um 
papel importante, sendo um local onde é possível promover mudanças na maneira de pensar e construir saúde em determinado contexto social (COSTA; SILVA; DINIZ, 2008).

O trabalho foi realizado na maior escola da América Latina, cuja população-alvo envolveu as crianças do $1^{\circ}$ e $2^{\circ}$ ano do ensino fundamental, que estudavam no período matutino. A peça teatral foi realizada em quatro momentos distintos, sendo dois momentos para os primeiros anos e outros dois para os segundos anos.

A escola possui pela manhã cinco turmas de $1^{\circ}$ ano, quatro turmas de $2^{\circ}$ ano, além de turmas do $3^{\circ}$ ao $5^{\circ}$ ano. O número de alunos por sala está distribuído da seguinte forma: $1^{\circ}$ ano até 24 alunos, $2^{\circ}$ ano até 28 alunos, $3^{\circ}$ ano até 32 alunos, $4^{\circ}$ até 32 alunos e $5^{\circ}$ até 35 alunos, contabilizando ao todo cerca de 1200 alunos. Os alunos com necessidades especiais contam com o auxílio de um segundo professor.

A atuação foi realizada em consonância com a demanda trazida pela escola e as ações propostas pela disciplina. Assim sendo, foram desenvolvidas ações de educação em saúde com a temática do bullying.

\section{Resultados e análises}

A atividade desenvolvida contou com a participação de 230 crianças e os professores de sala. A ação realizada foi uma das propostas de trabalho da equipe de alunos da fonoaudiologia, dado que na escola encontravam-se matriculadas crianças com múltiplas deficiências.

O bullying é um grande fator de risco para comportamentos antissociais individuais geradores de violência na sociedade (MAZINI; BRANCO, 2012). Neste sentido, pode trazer prejuízos à formação emocional, educacional e social da criança, contribuindo para problemas comportamentais e afetivos, como depressão, ansiedade, baixa autoestima, baixa auto aceitação, frustração e, em casos mais graves, suicídio (SALMIVALLI et al., 1996).

Neste contexto, a ação realizada corrobora com os aspectos instituídos pelo Programa de Combate à Intimidação Sistemática (Bullying) que considera bullying todo ato de violência física ou psicológica, intencional e repetitivo que ocorre sem motivação evidente, praticado por indivíduo ou grupo, contra uma ou mais pessoas, com o objetivo de intimidá-la(s) ou agredi-la(s), causando dor e angústia à vítima, em uma relação de desequilíbrio de poder entre as partes envolvidas. Tal programa aponta que é dever do estabelecimento de ensino assegurar medidas de conscientização, prevenção e combate ao bullying (BRASIL, 2015). 
Desta forma, os alunos do curso de fonoaudiologia abordaram a temática por meio de uma peça de teatro intitulada de "A qualidade das diferenças", elaborada pelos mesmos. A peça teve o intuito de promover a saúde e educação, uma vez que a mesma agregou personagens que possuíam algum tipo de dificuldade, fosse ela física ou intelectual.

A peça foi composta por seis personagens, os quais apresentavam características que foram selecionadas contemplando as necessidades da escola. Dentre os principais personagens estavam: Ligado (deficiente auditivo), Sorriso (deficiente físico) e Princesa (dificuldade de leitura e escrita) e outros que compuseram a peça, sendo eles: Soninho (realizava as atividades com lentidão), Gulosa (gostava de comer e estava acima do peso) e Belo (menino que se identificava com o gênero feminino).

O objetivo do teatro foi discutir a possibilidade de sensibilizar os alunos em relação às qualidades e dificuldades particulares de cada indivíduo assim como, o respeito às diferenças. As ações desenvolvidas corroboram com estudo brasileiro que trata do teatro como uma ferramenta de difusão do conhecimento que se quer tratar e de forma lúdica que ultrapassa a atividade de brincar, a criança passa a se interessar mais pelo conteúdo abordado e presta mais atenção no assunto apresentado (MIRANDA et al., 2009).

Nesse contexto encontram-se as ações de educação em saúde, ancoradas no conceito de promoção da saúde, que se vincula ao exercício da cidadania na busca por melhores condições de vida e de saúde da população, principalmente quando promovem espaços de troca de informação, diminuindo o distanciamento entre profissionais da saúde e população (OLIVEIRA et al., 2009).

Percebeu-se que a partir da atividade desenvolvida houve um retorno positivo das professoras e coordenadoras da escola que elogiaram o trabalho desenvolvido dando ênfase à temática tratada. Além disso, o retorno também foi observado pelos alunos que participaram e mostraram interesse pela história apresentada por meio de comentários e discussões realizadas ao final da peça.

A aliança entre saúde e educação aqui estabelecida, dá ênfase à intersetorialidade pontuada na Carta de Ottawa (OTTAWA, 1986), além de realçar que a promoção da saúde é considerada uma estratégia global incorporada na vida dos indivíduos, nos aspectos social, individual e ambiental e por isto não deve ser vista como responsabilidade exclusiva do setor de saúde, pois ela deve ser ampliada para além de um estilo de vida saudável, enfatizando as condições de vida e de trabalho dos sujeitos (MANTOVANI et al., 2014). 


\section{Considerações finais}

No presente trabalho pode-se observar a interação das crianças ao decorrer da peça de teatro e posteriormente a esta. As mesmas demonstraram interesse em falar sobre seus gestos de solidariedade ao outro, bem como, exemplificaram tais atitudes, contribuindo assim para o desfecho do teatro. Observou-se que a temática trabalhada despertou interesse dos alunos e professores. Os professores relataram que dariam continuidade no processo de educação em saúde na sala de aula, abordando o tema do teatro.

Ações como a peça de teatro, são importantes no âmbito escolar, pois despertam atenção da população infantil, promovendo respostas efetivas por serem realizadas de forma lúdica.

A promoção da saúde pode ser vista como um processo de capacitação da comunidade buscando a melhora de sua qualidade de vida. Esse processo ocorre por meio de uma modificação favorável no ambiente, após a identificação das necessidades em determinada área. Com isso, preconiza-se atingir o bem-estar físico, mental e social.

É fato que a ação buscou atingir a coletividade, no entanto, sabe-se que a ação foi pontual ao tratar-se de promoção da saúde, porém foi uma iniciativa que pode fomentar o processo de educação diante a parceria estabelecida com os educadores.

A saúde deve ser considerada um recurso para a vida, sendo um conceito positivo, enfatizando os recursos sociais e pessoais, assim como as capacidades físicas. Dessa forma, a promoção da saúde transcende o setor da saúde, relacionando-se ao bem-estar global do indivíduo.

\section{REFERÊNCIAS}

BEFI, D. A inserção da fonoaudiologia na atenção primária à saúde. In: BEFI, D. Fonoaudiologia na atenção primária à saúde. São Paulo: Lovise; 1997.

BRASIL. Ministério de Estado da Saúde. Portaria nº 2.488, de 21 de outubro de 2011a. Aprova a Política Nacional de Atenção Básica, estabelecendo a revisão de diretrizes e normas para a organização da Atenção Básica, para a Estratégia Saúde da Família (ESF) e o Programa de Agentes Comunitários de Saúde (PACS). Diário Oficial [da República Federativa do Brasil], 24 out. 2011; Seção 1, pt1. p.55.

BRASIL. Casa Civil. Decreto no 7.612, de 17 de novembro de 2011b. Institui o Plano Nacional dos Direitos da Pessoa com Deficiência - Plano Viver sem Limite. Disponível em: http://www.planalto.gov.br/ccivil_03/_Ato2011-2014/2011/Decreto/D7612.htm. Acesso em: 02 jun. 2016. 
BRASIL. Ministério da Saúde. Portaria no 2.446, de 21 de novembro de 2014. Redefine a Política Nacional de Promoção da Saúde (PNPS). Disponível em:

http://bvsms.saude.gov.br/bvs/saudelegis/gm/2014/prt2446_11_11_2014.html. Acesso em: 01 jun. 2016.

BRASIL. Casa Civil. Lei no 13.185, de 6 de novembro de 2015. Institui o Programa de Combate à Intimidação Siatemática (Bullying). Disponível em:

http://www.planalto.gov.br/ccivil_03/_Ato2015-2018/2015/Lei/L13185.htm. Acesso em: 02 jun. 2016.

COSTA, F. S.; SILVA, J. L. L.; DINIZ, M. I. G. A importância da interface educaçãolsaúde no ambiente escolar como prática de promoção da saúde. Informe-se em promoção da saúde, v. 4, n. 2, p. 30-3, 2008.

FEKKES, M.; PIJPERS, F. I.; VERLOOVE-VANHORICK, S. P. Bullying: who does what, when and where? Involvement of children, teachers and parents in bullying behavior. Health Educ Res, Oxford, v. 20, p. 81-91, fev. 2005.

FIGUEIREDO, T. A. M.; MACHADO, V. L. T.; ABREU, M. M. S. A saúde na escola: um breve resgate histórico. Ciênc Saúde Coletiva, Rio de Janeiro, v. 15, n. 2, p. 397-402, jan/mar. 2010.

JACOÉ, N. B.; et al. O olhar dos profissionais de uma Unidade Básica de Saúde sobre a implantação do Programa Saúde na Escola. Rev Méd Minas Gerais, Belo Horizonte, v. 24, n. 1, p. 43-48, (supl. 1) fev. 2014.

MALTA, D. C.; et al. Bullying in Brazilian schools: results from the National School-based Health Survey (PeNSE). Ciênc Saúde Coletiva, Rio de Janeiro, v. 15, n. 2, p. 3065-3076, out. 2010.

MANTOVANI, M. F.; et al. Representações sociais dos trabalhadores da estratégia saúde da família sobre promoção da saúde. Rev Enferm UFPE online, Recife, v. 8, n. 12, p. 42924299, dez. 2014.

MAZINI, R. G. P.; BRANCO, A. U. O bullying na perspectiva sociocultural construtivista. Bol Psicol., São Paulo, v. 62, n. 137, p. 169-182, dez. 2012

MIRANDA, J. L.; et al. Teatro e a escola: funções, importâncias e práticas. Revista CEPPG. Catalão, n. 20, p. 172-181. 2009.

OLIVEIRA, C. B.; et al. As ações de educação em saúde para crianças e adolescentes nas unidades básicas da região de Maruípe no município de Vitória Ciênc Saúde Colet, Rio de Janeiro, v. 14, n. 2, p. 635-644, abr. 2009.

OLIVEIRA, J. P.; NATAL, R. M. P. A linguagem escrita na perspectiva de educadores: subsídios para propostas de assessoria fonoaudiológica escolar. Rev CEFAC., São Paulo, v. 4, n. 6, p. 36-46, nov/dez. 2011. 
OTTAWA. Primeira Conferência Internacional sobre Promoção da Saúde. nov 1986. Disponível em: http://bvsms.saude.gov.br/bvs/publicacoes/carta_ottawa.pdf. Acesso em: 02 jun. 2016.

PEREIRA, D. L.; SANTOS, A. M. S.; OSBORN, E. Ação preventiva na escola: aspectos relacionados com a integração professor e aluno e a comunicação humana. In: VIERA, R. M.; et al. Fonoaudiologia e saúde pública. 2. ed. Carapicuíba: Pró-Fono; 2004.

SALMIVALLI, C.; et al. Bullying as group process: Participant roles and their relations to social status within the group. Aggressive Behaviour. v. 22, n. 1, p.1-15. 1996.

SANTOS, A. A. G.; et al. Sentidos atribuídos por profissionais à promoção da saúde do adolescente. Ciênc Saúde Coletiva, Rio de Janeiro, v. 17, n. 5, p. 1275-1284, maio. 2012.

VIEIRA, R. M.; et al. Fonoaudiologia e saúde pública. 2ed. Carapicuíba: Pró-Fono; 2004.

\section{Como referenciar este artigo}

PADILHA F. Y. O. M. M.; RODRIGUES, A. C. G.; SILVEIRA, I. C.; ARAKAWABELAUNDE, A. M. Fonoaudiologia e bullying: ação de promoção da saúde na escola. Revista Ibero-Americana de Estudos em Educação, Araraquara, v. 14, n. 2, p. 499-507, abr./jun., 2019. E-ISSN: 1982-5587. DOI: 10.21723/riaee.v14i2.8924

Submetido em: 29/02/2016

Revisões requeridas: $30 / 08 / 2016$

Aprovado em: 07/08/2017 